\title{
Modeling the Residual Energy and Lifetime of Energy Harvesting Sensor Nodes
}

\author{
M. Yousof Naderi, Stefano Basagni and Kaushik R. Chowdhury \\ Department of Electrical and Computer Engineering \\ Northeastern University, Boston, MA 02115, U.S.A. \\ E-mails: $\{$ naderi,basagni,krc\}@ece.neu.edu
}

\begin{abstract}
This paper presents SAVE, for Stochastic Analysis and aVailability of Energy, an analytical framework providing closed form expressions for residual energy and lifetime prediction of wireless sensor nodes. SAVE models a wide umbrella of input factors, including channel characteristics, different energy sources and harvesting policies, link layer parameters (e.g., error control and duty cycling) and various data traffic generation models. Our framework uses stochastic semi-Markov models to derive the residual energy distribution for each harvesting node accounting for practically observed temporal variations. We validate the analytical expressions derived by SAVE by means of simulations, and show that SAVE predictions provide a remarkably close match to the simulation results.
\end{abstract}

\section{INTRODUCTION}

Energy harvesting wireless sensor networks (EHWSNs) are made up of wireless sensor nodes powered by rechargeable batteries that are replenished through energy scavenged from renewable or ambient sources. Such a charging paradigm extends the network lifetime by reducing the charge drawn from the battery, prevents disruptions owing to battery replacement, and ensures environmentally friendly operation. However, as the residual energy at the node is time varying, and is subject to a variety of other factors, it is a challenge for the network designer to formulate closed form expressions that indicate future energy levels and lifetime of a EHWSN node. The analytical framework proposed in this paper, called Stochastic Analysis and aVailability of Energy (SAVE), takes the first step towards this direction.

Recent research on analyzing the lifetime and energy consumption in "classical," battery-limited WSNs has been presented in [1], [2], [3] (average analysis) and [4], [5] (distribution analysis). However, the key consideration of re-charging the energy reserve is not considered in these works. Seyedi et al. present a Markov-based model for energy harvesting nodes in a body sensor network [6]. The work provides an an analysis of the probability of event loss due to energy depletion. Moreover, Ventura and Chowdhury present MAKERS, a Markov based model for one or multiple-sources energy harvesting nodes in WSNs [7]. This work provides analytical model for predicting the

The research in this paper was supported in part by the NSF funded project "GENIUS: Green Sensor Networks for Air Quality Support" (NSF CNS 1143681). probability of a sensor running out of energy with very low computational complexity. In addition, it can model energy harvesting sensors equipped with multiple energy harvesting boards. Sus et al. present DTMC for environmentally powered sensor nodes for assessing statistical properties such as the probability of achieving a given operation time and the expected downtime [8]. While re-charging is considered, these models are examples of average analysis, where only the long term, average behavior of the nodes is ascertained, and do not consider the stochastic nature of energy availability.

SAVE is a general analytical framework that provides a stochastic tool for the analysis of the residual energy and the lifetime distribution of EHWSN nodes. We model the energy harvesting wireless sensor node (harvesting node, from now on) as a stochastic semi-Markov process and introduce a new analysis technique, called energy transient analysis, for the computation of the net consumed energy distribution. The amount of consumed and harvested energy in each state of a node are modeled as random variables, depending on discharging and recharging rates and on the holding time distribution of that state. The main contributions of this paper include the following: (i) We introduce SAVE for node residual energy and lifetime predictions at any time, based on the distributions of harvested energy, net-consumed energy (by processing, wireless re/transmissions, and sleep-awake cycles), and node lifetime. (ii) We show that SAVE is not limited to exponential or fixed holding time distributions in each Markov state. The operational states can have general and arbitrary holding time distributions, resulting in a more accurate and flexible analytical framework. (iii) We present a new technique called energy transient analysis serving as a tool for deriving the energy distributions based on the semi-Markov process of a harvesting node. This technique is harvesting technology- and harvesting node-independent, thus being applicable in a wide variety of practical settings.

\section{The SAVE ANALYTICAL FramEWORK}

We consider nodes equipped with one or multiple energy harvesting modules, working under different energy sources, each with their independent charging and discharging rates. SAVE models a harvesting sensor node as a semiMarkov process (SMP) in which each state corresponds to 
a node operational mode. A SMP is a type of stochastic process whose states change as in a Markov chain, but where the permanence in a particular state happens for a random amount of the time (called holding time), following a given distribution. The holding time depends on the current state and on the next state to be visited. In a SMP, each state is associated with one or more reward variables called reward rates. In the case of SAVE, every state $i$ has two reward rates: Power discharging (battery consumption) rate $d_{i}$ and power recharging (energy replenishment) rate $r_{i}$. The discharging rate depends on the node hardware specifications and on the node operation of that state. The power recharging rate depends on the renewable energy source.

With $X_{h}=\{X(t): t \geq 0\}$ we represents the SMP of the harvesting node $h$ with sample states $S=\{1,2, . ., M\}, M$ being the number of a harvesting node operational states. The random variable $X(t)$ indicates the state of the system at time $t$. The Markov renewal process $Z=\left\{\left(Z_{n}, T_{n}\right) ; n \geq\right.$ $0\}$ is connected to the SMP $X_{h}$ as follows:

$$
X(t)=Z_{n}, T_{n} \leq t<T_{n+1},
$$

where $n$ is the number of state transitions that has taken place by time $t, Z_{n} \in S$ is the state after the $n^{t h}$ transition and $T_{n}$ is the time of the $n^{\text {th }}$ transition. The state transition probability $P_{i j}$, which governs the change from state $i$ to state $j$, is defined as follows:

$$
P_{i j}=\operatorname{Pr}\left\{X\left(T_{1}\right)=j \mid X(0)=i\right\} .
$$

Accordingly, $P=\left[p_{i j}\right]$ is the transition probability matrix. The kernel of the semi-Markov process is independent of the number $n \geq 0$ of transitions, and it is defined as:

$$
K(i, j, t)=\operatorname{Pr}\left(Z_{n+1}=j, T_{n+1}-T_{n} \leq t \mid Z_{n}=i\right),
$$

or, equivalently:

$$
K(i, j, t)=P_{i j} Q_{i j}(t) .
$$

In the latter equation $Q_{i j}(t)$ denotes the holding time distribution of state $i$, after a transition from state $i$ to state $j$ has occurred. More specifically:

$$
Q_{i j}(t)=\operatorname{Pr}\left(T_{n+1}-T_{n} \leq t \mid Z_{n+1}=j, Z_{n}=i\right) .
$$

The state transition probabilities and holding time distributions depend on the specific network and protocol parameters.

The matrix $K(t)=[K(i, j, t)]$ is the kernel matrix of the harvesting node and stores information on the harvesting node operations. This is the information we need for computing the energy and node lifetime distributions. In other words, the stochastic nature of the harvesting node is captured by the kernel of its SMP. The distribution of the holding time in a particular state $i$ is obtained from the kernel matrix of the SMP as follows:

$$
Q_{i}(t)=\sum_{j \in S} K(i, j, t)
$$

Fig. 1 shows two generic states of the SMP representing a harvesting node. The time-varying behavior of a harvesting node from state $i$ to state $j$ is expressed by the transition probability $P_{i j}$ and the holding time distribution $Q_{i j}(t)$, while the power discharging and recharging rates capturing the consumed and harvested energies.

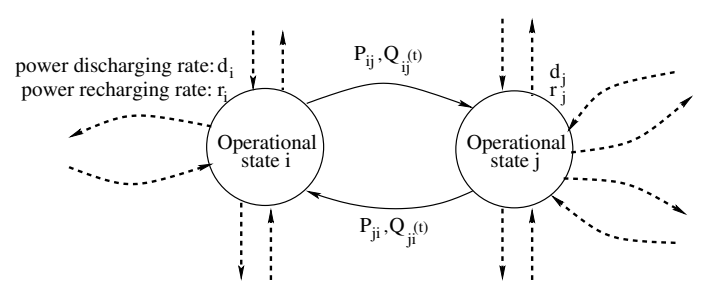

Fig. 1: A harvesting node as a semi-Markov process.

\section{A. Residual energy distribution}

The amount of residual energy at a harvesting nodes over time plays a key role in fundamental network functions, such as its connectivity, coverage and lifetime. In this section, we derive the distribution of the energy available at a harvesting node at a given time $T$.

The amount of energy consumed in state $i$ is a random variable $E_{i}$ that depends on the discharging rate $d_{i}$ and on the probability distribution of the holding time in that state. Similarly, the amount of harvested energy in state $i$ is a random variable $H_{i}$ that depends on the recharging rate $r_{i}$ and the holding time distribution. The energy consumption distribution in state $i$ when a transition from state $i$ to state $j$ occurs, is as follows:

$$
E_{i j}(e)=\operatorname{Pr}\left(E_{i} \leq e \mid Z_{n+1}=j, Z_{n}=i\right) .
$$

For example, if the discharging rate in state $i$ is $d_{i}$ and $Q_{i j}(t)$ is the holding time distribution before the harvesting node moves into state $j$, the energy consumption distribution $E_{i j}(e)$ is:

$$
E_{i j}(e)=Q_{i j}\left(\frac{e}{d_{i}}\right) .
$$

Similarly, the energy harvesting distribution in state $i$ when SMP moved from state $i$ to $j$ is:

$$
H_{i j}(e)=\operatorname{Pr}\left(H_{i} \leq e \mid Z_{n+1}=j, Z_{n}=i\right) .
$$

The unconditional energy consumption and harvesting distributions in state $i$ when a transitions form state $i$ to state $j$ occurs are:

$$
E(i, j, e)=\operatorname{Pr}\left(Z_{n+1}=j, E_{i} \leq e \mid Z_{n}=i\right)=P_{i j} E_{i j}(e)
$$

and:

$H(i, j, e)=\operatorname{Pr}\left(Z_{n+1}=j, H_{i} \leq e \mid Z_{n}=i\right)=P_{i j} H_{i j}(e)$, 
Clearly, considering the discharging and recharging rates, both of the two last distributions could be computed based on the kernel of the semi-Markov process. The distribution of energy consumption and harvesting in state $i$ are finally obtained as follows:

$$
E(i, e)=\sum_{j \in S} E(i, j, e) \text { and } H(i, e)=\sum_{j \in S} H(i, j, e) .
$$

We now describe a new energy transient analysis technique for computing the distributions of the consumed and of the harvested energy. We assume that the harvesting node starts in state $i$ and after time $T$ is in state $k$. Throughout time $T$ the node might have transitioned through different sequences of states (paths in the SMP). However, independently of the followed path, for each state $m$ in the path the harvesting node consumes a random amount of energy $E_{m}$ and harvests a random amount of energy $H_{m}$. Both random values $E_{m}$ and $H_{m}$ depend on the state holding time probability distribution, and on the discharging and recharging rates associated to state $m$. Therefore, the total amount of energy consumed and harvested over a path of $N$ states from state $i$ to state $k$ during time $T$ can be obtained as the sum of $N$ independent random variables:

$$
\begin{gathered}
E_{i k}(T)=E_{i}+E_{i+1}+\ldots+E_{m}+\ldots+E_{k}, \text { and } \\
H_{i k}(T)=H_{i}+H_{i+1}+\ldots+H_{m}+\ldots+H_{k},
\end{gathered}
$$

where $k=N+i-1$. The probability density function (pdf) $f_{i k}^{e}(e, T)$ of $E_{i k}$ is defined such that:

$$
\operatorname{Pr}\left(e_{1} \leq E_{i k} \leq e_{2}\right)=\int_{e_{1}}^{e_{2}} f_{i k}^{e}(e, T) .
$$

The pdf $f_{i k}^{h}(e, T)$ of $H_{i k}$ is defined similarly. Recall that the pdf of the sum of $N$ independent random variables is the $N$-fold convolution of their pdfs. Therefore, in order to determine the joint conditional pdf of energy consumption (conditioned with respect to time $T$ and initial state $i$ ) it is necessary to convolve the energy consumption pdfs of the states, $\frac{d E_{i j}(e)}{d e}$, over all possible paths starting from state $i$ and finishing in state $k$ throughout time $T$. To this end, we calculate the Laplace transform of the convolutions over all possible paths with different state holding times. This is done through the following theorem.

Theorem 1: For any given time $T$, the Laplace transform $L_{i k}^{e}(s, T)$ of $f_{i k}^{e}(e, T)$ with respect to $e$, is the solution of the following equation:

$$
\begin{aligned}
& L_{i k}^{e}(s, T)=e^{-s d_{i} T}\left(1-E\left(i, d_{i} T\right)\right) \\
& \quad+\sum_{m} \int_{0}^{T d_{i}} e^{-s e} L_{m k}^{e}\left(s, T-\frac{e}{d_{i}}\right) d E(i, m, e),
\end{aligned}
$$

where $m$ ranges over all states in the $\operatorname{SMP} X$, and $d_{i}$ is the discharging rate in state $i$.
Proof: Let $E$ be a random variable with cumulative distribution function (CDF) F(e). The Laplace-Stieltjes transform (LST) of F is defined as follows:

$$
E\left(e^{-s E} ; E \leq e \mid Z_{0}=i\right)=\int_{0}^{\infty} e^{-s e} d F(e) .
$$

The Laplace transform of $f_{i k}^{e}(e, T)$ is as follows:

$$
L_{i k}^{e}(s, T)=E\left(e^{-s E_{i k}(T)} ; E_{i k}(T) \leq e \mid Z_{0}=i\right),
$$

where the random variable $E_{i k}(T)$ shows the total amount of consumed energy during time $T$ starting from state $i$. Conditioning on the initial state energy consumption $E_{i}$ we have:

$$
\begin{array}{r}
E\left(e^{-s E_{i k}(T)} ; E_{i k}(T) \leq e \mid E_{i}=c, Z_{0}=i\right)= \\
\begin{cases}e^{-s d_{i} T} & c \geq d_{i} T, \\
e^{-s c} \sum_{m} L_{m k}^{e}\left(s, T-\frac{c}{d_{i}}\right) & c<d_{i} T .\end{cases}
\end{array}
$$

Now, unconditioning with respect to $E_{i}$, we obtain:

$$
\begin{aligned}
& L_{i k}^{e}(s, T)= \\
& \quad \int_{0}^{T d_{i}} e^{-s e} \sum_{m} L_{m k}^{e}\left(s, T-\frac{e}{d_{i}}\right) d E(i, m, e) \\
& \quad+e^{-s d_{i} T}\left(1-E\left(i, d_{i} T\right)\right)= \\
& \sum_{m} \int_{0}^{T d_{i}} e^{-s e} L_{m k}^{e}\left(s, T-\frac{e}{d_{i}}\right) d E(i, m, e) \\
& \quad+e^{-s d_{i} T}\left(1-E\left(i, d_{i} T\right)\right) .
\end{aligned}
$$

Similarly, $L_{i k}^{h}(s, T)$ is calculated as follows:

$$
\begin{aligned}
& L_{i k}^{h}(s, T)= \\
& \quad \sum_{m} \int_{0}^{T r_{i}} e^{-s e} L_{m k}^{h}\left(s, T-\frac{e}{r_{i}}\right) d H(i, m, e) \\
& \quad+e^{-s r_{i} T}\left(1-H\left(i, r_{i} T\right)\right),
\end{aligned}
$$

where $r_{i}$ is the recharging rate in state $i$. The energy available at the harvesting node at time $T$ is defined as the residual battery energy taking into account the net consumed energy until time $T$. The net consumed energy includes both consumed and harvested energies. Since the consumption process is stochastically independent of the harvesting process, we can derive the Laplace transform of the net consumed energy during time $T$ as

$$
L_{i}(s, T)=L_{i k}^{e}(s, T) L_{i k}^{h}(s, T),
$$

Thus, the pdf $f(e, T)$ of the net consumed energy $e$ consumed through time $T$ can be calculated by numerically inverting the Laplace transform $L_{i}(s, T)$. Then, the $\mathrm{CDF} E(e, T)$ is found by $\int_{0}^{e} f(e, T) d e$. Note that such distribution depends on the initial state $i$, which in this 
work is assumed to be always the sleep state. Finally, the distribution of the energy available to harvesting node, residual energy, after time $T$ is given by:

$$
P^{A}(e, T)=E\left(E_{0}-e, T\right),
$$

which depends on the overall net consumed energy distribution $E(e, T)$ and the initial energy of the primary battery $E_{0}$. The set of transitions from one specific state (e.g., sleep state) to the next visit to that state construct a harvesting node operational cycle. As a special case, the energy distributions regarding the number of cycles can be found based on the steady-state probabilities of the SMP, $\pi_{i}$, and the average time spent in each state, $\mu_{j}$. In particular, the average length of one cycle is equal to $\sum_{j} \pi_{j} \mu_{j}$, and accordingly one can compute the Laplace transform of the net consumed energy and its equivalence residual energy for different number of cycles.

\section{B. Node lifetime distribution}

In this section, considering the sensor semi-Markov model $X(t)$, we characterize the harvesting node lifetime distribution as a function of time (or number of cycles). Let $g$ be the power consumption reward function which for any time $t$ over the operation of the sensor defined as:

$$
g(X(t))=d_{i} \geq 0, \text { if } X(t)=i,
$$

where $g(X(t))$ indicates the power consumption per unit time in the state $i$ (at time t). Similarly, we consider $f$ to be the charging reward function as:

$$
f(X(t))=r_{i} \geq 0, \text { if } X(t)=i,
$$

$r_{i}$ represents the energy recharging rate of the harvesting sensor in state $i$. We define the net consumed energy $E(t)$ as a random variable which takes into consideration both accumulated charging and discharging energies of a harvesting sensor node in time as follows:

$$
\begin{aligned}
E(t) & =\int_{0}^{t}[g(X(\tau))-f(X(\tau))] d \tau \\
& =\int_{0}^{t}\left[d_{X(\tau)}-r_{X(\tau)}\right] d \tau=\int_{0}^{t} e_{X(\tau)} d \tau .
\end{aligned}
$$

where $e_{i}$ indicates the combined reward rate in state $i$ when $X(t)=i$ and $E(t)$ is a stochastic process based on $X(\tau)$ for $0<\tau<t$. The harvesting sensor node lifetime can be defined as a random variable $T$ representing the time to accumulate a combined reward requirement equal to a random variable $w$ which represents the initial energy of the sensor's battery $\left(w=E_{0}\right)$ :

$$
T(w)=\min \{t \geq 0: E(t)=w\},
$$

Moreover, node lifetime $T$ is the time instant at which the overall (net) consumed energy by the sensor node reaches the value $w$ for the first time. Given that the initial state is $i$, the probability that harvesting node consumes more
TABLE I: Parameters of SAVE Framework for the Case Study

\begin{tabular}{l||l}
\hline Parameter & Meaning \\
\hline \hline$E_{0}$ & Initial energy of the on-board battery \\
$G$ & Maximum number of retransmissions \\
$B E R$ & Channel bit error rate \\
$P_{e}$ & Packet error rate \\
$P_{B}$ & Channel busy probability \\
$K$ & Maximum number of backoff occurrences \\
$T_{s}$ & Sleeping period \\
$T_{w}$ & Wakeup period \\
$\lambda^{e}$ & Average event arrival rate \\
$S_{p}$ & Data packet size \\
$S_{M}$ & Data message size \\
$S_{H}$ & Packet header size \\
$R_{t x}$ & Channel transmission rate \\
$t_{c h}$ & Average charging time \\
$P_{s c}$ & Charging probability \\
$B$ & Average processing time per event \\
$P_{I}$ & Event of interest probability \\
\hline \multicolumn{2}{|}{}
\end{tabular}

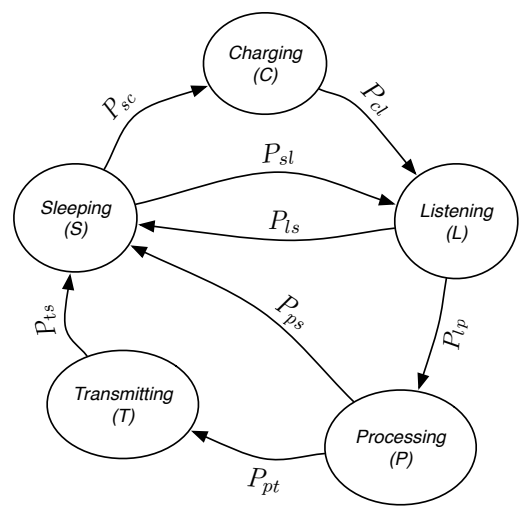

Fig. 2: Semi-Markov Chain of a Harvesting Sensor Node

accumulated energy than $w$ during sensor operation time $t$ is :

$$
\operatorname{Pr}(E(t)>w \mid X(0)=i),
$$

which is exactly the probability that the lifetime of the harvesting node is shorter than $t$. Correspondingly, the conditional node lifetime distribution which is the probability distribution that the sensor node achieves the desired lifetime $t$, when $i$ is the given initial state, would be as :

$$
\operatorname{Pr}(T(w) \leq t \mid X(0)=i)=\operatorname{Pr}\left(E(t)>E_{0} \mid X(0)=i\right),
$$

\section{Framework ANALysis: A CASE STUdy}

In section II, we described a general analysis framework for any arbitrary state diagram that defined the operation of an energy harvesting sensor. In this section, we take a specific example of a sleep-awake duty cycled sensor that harvests energy with an exponential charging time, and uses a CSMA-based MAC protocol with ARQ for error recovery at the link layer. A detailed description of the variables used is given in Table I. 


\section{A. Semi-Markov chain overview}

The functions of the harvesting sensor node is modeled as a semi-Markov chain consisting of (i) charging (C), (ii) sleeping (S), (iii) listening (L), (iv) processing (P), and (v) transmitting $(\mathrm{T})$ operational states, which are represented in Fig. 2 with their corresponding abbreviations in the parenthesis. The operation of the sensor for this example scenario is described in the following, assuming the above states:

1) Energy Harvesting Policy: The initial energy of the primary battery is $E_{0}$. During its reduced activity sleep time (i.e., in state $\mathrm{S}$ ), the sensor may either transition into deep sleep and charge (i.e., enter state $\mathrm{C}$ ) with the probability $P_{s c}$, which is equal to a given charging probability, for an exponentially distributed time with the mean value of $\overline{t_{c h}}$, or continue in its current state with probability $1-P_{s c}$ for a fixed sleeping period $T_{s}$. Note that while our framework supports any arbitrary harvesting policy, for the purpose of tractability, we assume here an exponential charging time. After completing the sleeping period $T_{s}$, the node switches to the listening state (i.e., state L) and starts sensing the environment.

2) Event Processing: The node returns back to the sleeping state when no event is detected, or else it processes the detected events in the processing state (i.e., state P) to find out if any of them are the events of interest. The processing time per event follows an exponential distribution with mean $B$, though our general model supports arbitrary distributions. Consequently, the processing time of $R$ events has R-stage Erlang distribution.

3) MAC Protocol: After completion of the processing stage, if any desired event is detected, the node switches to the transmitting state (i.e., state $\mathrm{T}$ ) with probability $P_{p t}$, which is derived later in Section III-B. If the sensor node finds the channel idle during its initial carrier sensing, it begins transmitting. But if the channel is busy with probability $P_{B}$, the node defers its transmission for a random amount of time. It waits in the idle mode, and probes the wireless channel again after that time. A maximum number of $K$ backoff occurrences for each packet is assumed. Moreover, the path loss wireless channel is modeled with a two-state discrete Markov chain known as Gilbert model in which $P_{e}$ indicates the average packet error probability and is computed based on the wireless channel bit error rate. Finally, an ARQ error control mechanism with $G$ maximum number of packet retransmissions is considered.

\section{B. Constructing the SMP kernel}

In this section, we construct the kernel matrix of our example. In particular, each kernel has two elements: probability transition matrix $(\mathrm{P})$ and holding time distribution matrix (Q). Next, we derive the transition probabilities and holding time distributions of our SMP described in Figure 2. Note that the matrix elements for non-existent transition paths in SMP are zero.

$$
\begin{gathered}
P=\left(\begin{array}{ccccc}
P_{s s} & P_{s c} & P_{s l} & \cdots & P_{s t} \\
P_{c s} & P_{c c} & P_{c l} & \cdots & P_{c t} \\
\vdots & \vdots & \vdots & \ddots & \vdots \\
P_{p s} & P_{p c} & P_{p l} & \cdots & P_{p t} \\
P_{t s} & P_{t c} & P_{t l} & \cdots & P_{t s}
\end{array}\right) \\
Q=\left(\begin{array}{ccccc}
Q_{s s}(t) & Q_{s c}(t) & Q_{s l}(t) & \cdots & Q_{s t}(t) \\
Q_{c s}(t) & Q_{c c}(t) & Q_{c l}(t) & \cdots & Q_{c t}(t) \\
\vdots & \vdots & \vdots & \ddots & \vdots \\
Q_{p s}(t) & Q_{p c}(t) & Q_{p l}(t) & \cdots & Q_{p t}(t) \\
Q_{t s}(t) & Q_{t c}(t) & Q_{t l}(t) & \cdots & Q_{t t}(t)
\end{array}\right)
\end{gathered}
$$

The holding time distributions of sleeping state is $Q_{s l}(t)=$ $U\left(t-T_{s}\right)$ and $Q_{s c}(t)=0$, here $U$ is the unit step function. Furthermore, the holding time in charging state has exponential distribution $Q_{c l}(t)=1-e^{-\frac{t}{\overline{t_{c h}}}}$. Since the event arrivals occur during the awake period follow a Poisson process with average rate $\lambda^{e}$, the transition probabilities of listening state are $P_{l s}=e^{-\lambda^{e} T_{w}}$ and $P_{l p}=1-e^{-\lambda^{e} T_{w}}$, and staying time distribution is $Q_{l s}(t)=Q_{l p}(t)=U\left(t-T_{w}\right)$.

Assuming $N_{E}^{l}$ is the number of received events during listening time, then the probability that $n$ events are detected and the average number of sensed events are:

$$
\begin{gathered}
f_{e}^{l}(n)=\operatorname{Pr}\left(N_{E}^{l}=n\right)=\frac{\left(\lambda^{e} T_{w}\right)^{n} e^{-\lambda^{e} T_{w}}}{n !}, \\
E\left[N_{E}^{l}\right]=\lambda^{e} T_{w} .
\end{gathered}
$$

Additionally, when $n$ events are detected during the listening time, the total summation of the exponential processing time approaches the n-stage Erlang distribution with density function $f_{\text {Erlang }}(n, B, t)$ with the cumulative distribution $H_{\text {Erlang }}(n, B, t)$. The probability that the processing state reveals no event of interest is equal to transition probability $P_{p s}$, and is calculated by:

$$
P_{p s}=\sum_{n=0}^{\infty} f_{e}^{l}(n)\left(1-P_{I}\right)^{n} .
$$

Here, $P_{I}$ is the probability of a single event being of interest. We approximate $P_{p s}=\left(1-P_{I}\right)^{E\left[N_{E}^{l}\right]}$ with the average number of detected events, without a significant loss of precision. Similarly, the distribution of the holding time at the processing state can be computed by using the k-stage Erlang distribution, with the average value $E\left[N_{E}^{l}\right]$ as follows:

$$
Q_{p s}(t)=Q_{p t}(t)=H_{\text {Erlang }}\left(\left\lceil E\left[N_{E}^{l}\right]\right\rceil, B, t\right) .
$$

Let $N_{E}^{T}$ indicate the number of events of interest detected after processing, having the average value equal to $\lambda^{e} T_{w} P_{I}$. Thus, the probability of detecting $i$ events of interest is:

$$
g_{e}^{t}(i)=\operatorname{Pr}\left(N_{E}^{T}=i\right)=\sum_{n=0}^{\infty} f_{e}^{l}(n)\left(\begin{array}{c}
n \\
i
\end{array}\right)\left(P_{I}\right)^{i}\left(1-P_{I}\right)^{n-i} .
$$


Considering the average number of desired events, this probability is approximated by:

$$
g_{e}^{t}(i)=\operatorname{Pr}\left(N_{E}^{T}=i\right)=\left(\begin{array}{c}
\lambda^{e} T_{w} \\
i
\end{array}\right)\left(P_{I}\right)^{i}\left(1-P_{I}\right)^{\lambda^{e} T_{w}-i} .
$$

The transition probability from transmitting state to sleeping state is equal to one. Furthermore, the staying time at the transmitting state is related to the back-off time, packet transmission time, wireless channel error probability, and maximum allowable number of retransmissions. Accordingly, we first compute the holding time distribution of transmitting one packet and then extend it to the total number of packets sent in the burst (i.e., in state $\mathrm{T}$ of the current cycle).

Let $T_{T}^{P}$ be holding time for one packet transmission in the state $T$. Then $T_{T}^{P}$ and its pdf $f_{t}^{p}(t)$ can be represented as follows:

$$
\begin{gathered}
T_{T}^{P}=T_{\text {backoff }}+T_{T x}, \\
f_{t}^{p}(t)=f_{\text {backoff }}(t) * f_{T x}(t),
\end{gathered}
$$

where * represents a convolution operation. Accordingly, to compute the $f_{t}^{p}(t)$, we need to find the probability density of back-off time as well as the probability density of a packet transmission time. Clearly, as the transmission time of a packet $T_{\text {packet }}$ is a constant value, $f_{T x}(t)$ is:

$$
\begin{gathered}
f_{T x}(t)=\delta\left(t-T_{\text {packet }}\right), \\
T_{\text {packet }}=\frac{S_{\text {packet }}+S_{\text {header }}}{R_{t x}},
\end{gathered}
$$

which based on the convolution property of the delta function results in $f_{t}^{p}(t)=f_{\text {backof } f}\left(t-T_{\text {packet }}\right)$. Next, assume $X_{i^{\prime}}$ is a random variable that indicates the backoff time of $i^{\prime}+1$-th attempt and $Y_{i^{\prime}}$ is random variable of the total (sum) backoff time for $i^{\prime}+1$ backoff attempts. The probability density function of $Y_{i^{\prime}}$ can be calculated based on the pdf of $X_{i^{\prime}}$ as shown:

$$
\begin{gathered}
f_{Y_{m}}(t)=f_{X_{0}}(t) * f_{X_{1}}(t) * \ldots . . * f_{X_{m}}(t), \\
f_{Y_{m}}(t)=f_{Y}(m, t)=f_{X_{m}}(t) * f_{Y}(m-1, t),
\end{gathered}
$$

where:

$$
f_{X_{i}}(t)=U\left(0,2^{i+1}\right)= \begin{cases}\frac{1}{2^{i+1}} & 0 \leq t \leq 2^{i+1}, \\ 0 & t>2^{i+1} \text { or } t<0 .\end{cases}
$$

Consequently, the probability density function of total backoff time for one packet transmission would be determined as follows:

$$
\begin{array}{r}
f_{\text {backoff } f}(t)=f_{Y_{0}}(t) P_{0}+f_{Y_{1}}(t) P_{1}+\ldots \ldots \\
\ldots \ldots .+f_{Y_{K-1}}(t) P_{K-1}+f_{Y_{K}}(t) P_{K} \\
P_{i}= \begin{cases}\left(P_{B}\right)^{i}\left(1-P_{B}\right) & 0 \leq i \leq K-1 \\
\left(P_{B}\right)^{i} & i=K\end{cases}
\end{array}
$$

where $P_{B}$ is the probability that the sensed channel is busy before transmission and where $K$ is the maximum number of allowable back-offs. Then the pdf of holding time in state $T$ for one packet transmission (Equation 3) can be computed based on the equations 4 and 5 .

The above analysis considers a single packet transmission. However, the wireless channel introduces an average packet error rate $P_{e}$ and ARQ error recovery permits $G$ number of packet retransmissions. Moreover, for each detected event of interest, the sensor node packs its sensed information into a data message and transmits to the base station. Therefore, there are a number of packets sent per desired event, depending on the size of packet and data message, that is calculated as $\alpha=\left\lceil\frac{S_{M}}{S_{P}}\right\rceil$, where $S_{M}$ and $S_{P}$ represent the sizes of the data message and packet, respectively. Accordingly, next we extend this analysis for the case of multiple packet transmissions that result from wireless channel errors and the message.

The expected number of transmissions for one packet $\mathrm{E}[\gamma]$ until it is successfully transmitted or dropped is computed as follows:

$$
\begin{gathered}
f_{\gamma}(i)= \begin{cases}\left(P_{e}\right)^{i-1}\left(1-P_{e}\right) & 1 \leq i<G, \\
\left(P_{e}\right)^{i} & i=G .\end{cases} \\
E[\gamma]=\sum_{i=1}^{G}(i) f_{\gamma}(i)=\sum_{i=1}^{G-1}\left[i\left(P_{e}\right)^{i-1}\left(1-P_{e}\right)\right] \\
+G\left(P_{e}\right)^{G}=\frac{1-\left(p_{e}\right)^{G}}{1-P_{e}} .
\end{gathered}
$$

where $\gamma$ is a random variable of the number of transmissions per packet. The average number $E\left[N^{T}\right]$ of total transmitted packets $N^{T}$ (also considering retransmissions) in the state $T$ in the current cycle is:

$$
E\left[N^{T}\right]=(\alpha) E[\gamma] E\left[N_{E}^{T}\right] .
$$

The probability density function of the holding time of the transmitting state can be derived by the using equations 2,3 , and 6 as follows:

$$
\begin{gathered}
f_{t s}(t)=\sum_{j=1}^{\infty} g_{e}^{t}\left(\left\lfloor\frac{j}{\alpha}\right\rfloor\right) G_{t}(j E[\gamma], t), \\
G_{t}(n, t)=f_{t}^{p}(t) * G_{t}(n-1, t),
\end{gathered}
$$

which is also approximated by $f_{t s}(t)=G_{t}\left(E\left[N^{T}\right], t\right)$. Then, the holding time distribution at state $T$ is computed from the density function by integrating $f_{t s}(t)$ from 0 to $t$.

\section{Performance Evaluation}

In this section, we present the simulation and analytical results of the energy and lifetime distributions evaluated through MATLAB. The packet data size is 140 bytes, with the data and header sizes being $S_{p}=128$ bytes and $S_{h}=12$ bytes, respectively. The data message size $S_{M}$ is set to 1 kbyte. The event arrival rate is $\lambda^{e}=0.15$. The wireless channel bit error rate (BER) is $10^{-3}$, and 


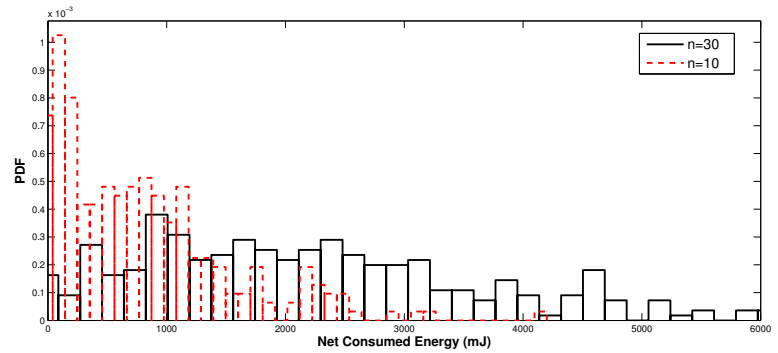

(a) simulation results

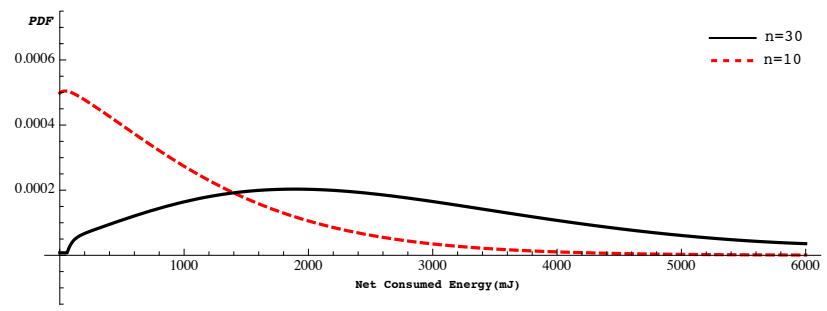

(b) analytical results

Fig. 3: Probability density functions of net consumed energy for different operational cycles $n=10$ and $n=30$.

channel busy probability is set to $P_{B}=0.2$. The packets are transmitted with a rate $250 \mathrm{Kbps}$ and the maximum number of retransmissions $G=3$. Also, a CSMA-based MAC with maximum five allowable backoff occurrences is employed. The sleeping and wakeup periods are simulated with $T_{s}=0.2 \mathrm{~s}$ and $T_{w}=1.8 \mathrm{~s}$, respectively. For the harvesting parameters, the charging probability is equal to 0.3 and the average charging time is set as $2 \mathrm{~s}, \overline{t_{c h}}=2$. Each packet or event would be processed for an average of $0.2 \mathrm{~s}$, while the probability of the event being of interest is 0.8 . The energy consumption rates are set based on the Imote2 sensor mote [1]. More specifically, the discharging rates for sleeping, listening, processing, and transmitting are set to $1.8,88,237,273 \mathrm{~mW}$, respectively, and recharging rate is set to $50 \mathrm{~mW}$. The simulations are performed 200 times for each node with different random number seeds. Fig. 3(a) shows the simulation and Fig. 3(b) presents the corresponding analytical results of net consumed energy after 10 and 30 cycles. It is shown that in both of simulation and analytical analysis, the density of net consumed energy is converging to an asymptotic Normal distribution. Moreover, comparing the density functions of simulation and analytical results in Fig. 3, the accuracy of the analysis in predicting the probability of net consumed energy can be observed. Clearly, by knowing the net consumed and initial energy, the residual energy is determined by using Equation 1. However, due to space limitations, here, we have presented only the net consumed energy distributions. It worth to notice that since the number of simulation runs are finite, the histogram (pdf) of net consumed energy is computed by employing discretization and bins.

Since the node lifetime is defined as the time until the residual energy drops below a minimum energy requirement, the initial energy of the on-board primary battery directly affects the distribution of lifetime. Fig. 4 depicts results for node lifetime, analysis and simulation, with $E_{0}=10$ Joules. We observe an accurate match between the simulated results for node lifetime and the analytical model derived in this paper.

\section{CONClusion}

In this paper, we proposed a generic framework for analysis of energy and lifetime distributions in energy har-

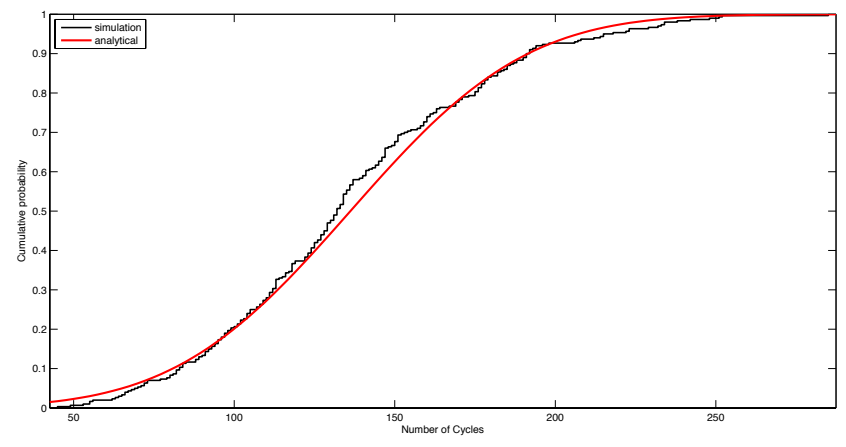

Fig. 4: Distribution of node lifetime with $E_{0}=10$ Joules.

vesting sensor nodes. The behavior of a harvesting sensor node is modeled by a semi-Markov model, in which the discharging and recharging rates are assumed as the semiMarkov state reward rates. The node lifetime is calculated through the energy transient analysis approach. Finally, the simulation and analytical results for net consumed energy and node lifetime distributions reveal a close match thereby verifying the correctness of our approach.

\section{REFERENCES}

[1] D. Jung, T. Teixeira, and A. Savvides, "Sensor node lifetime analysis: Models and tools," ACM Transactions on Sensor Networks, vol. 5, pp. 3:1-3:33, February 2009.

[2] R. A. F. Mini, M. do Val Machado, A. A. F. Loureiro, and B. Nath, "Prediction-based energy map for wireless sensor networks," Ad Hoc Networks, vol. 3, pp. 235-253, March 2005.

[3] S. Coleri, M. Ergen, and T. J. Koo, "Lifetime analysis of a sensor network with hybrid automata modeling," in Proceedings of the ACM WSNA 2002, Atlanta, Georgia, September 2002, pp. 98-104.

[4] Y. Wang, M. C. Vuran, and S. Goddard, "Stochastic analysis of energy consumption in wireless sensor networks," in Proceedings of IEEE SECON 2010, June 2010, pp. 1-9.

[5] M. Noori and M. Ardakani, "Lifetime analysis of random event-driven clustered wireless sensor networks," IEEE Transactions on Mobile Computing, vol. 10, no. 10, pp. 1448-1458, October 2011.

[6] A. Seyedi and B. Sikdar, "Modeling and analysis of energy harvesting nodes in wireless sensor networks," in Proceedings of the 46th Allerton Conference on Communication, Control, and Computing, September 2008, pp. 67-71.

[7] J. Ventura and K. R. Chowdhury, "Markov modeling of energy harvesting body sensor networks," in Proceedings of IEEE PIMRC 2011, September 2011, pp. 2168-2172.

[8] A. E. Susu, A. Acquaviva, D. Atienza, and G. De Micheli, "Stochastic modeling and analysis for environmentally powered wireless sensor nodes," in Proceedings of 6th International Symposium on Modeling and Optimization in Mobile, Ad Hoc, and Wireless Networks, WiOpt 2008, April 2008, pp. 125-134. 